\title{
Differences in Photosynthesis of Variegated Temple Bamboo Leaves with Various Levels of Variegation are Related to Chlorophyll Biosynthesis and Chloroplast Development
}

\author{
Lingyan Chen, Jinli Lai, and Tianyou He \\ College of Arts \& College of Landscape Architecture, Fujian Agriculture and Forestry University, \\ Fuzhou, Fujian 350002, People's Republic of China \\ Jundong Rong and Muhammad Waqqas Khan Tarin \\ College of Forestry, Fujian Agriculture and Forestry University, Fuzhou, Fujian 350002, People's \\ Republic of China \\ Yushan Zheng ${ }^{1}$ \\ College of Arts \& College of Landscape Architecture, Fujian Agriculture and Forestry University, \\ Fuzhou, Fujian 350002, People's Republic of China
}

\begin{abstract}
Additional INDEX WORDs. chlorophyll biosynthesis precursors, chloroplast ultrastructure, photosynthesis curve
Abstract. Variegated temple bamboo (Sinobambusa tootsik f. luteoloalbostriata) is a species of ornamental bamboo (Bambusoideae) that has gained popularity because of its striped or variegated leaves. In this study, a series of experiments was conducted to determine the factors contributing to the leaf color of this species, which included the content of the photosynthetic pigments and the chlorophyll biosynthetic precursors, the photosynthetic parameters, and the microstructure and ultrastructure of the different phenotypes. Discoloration in the leaves of variegated temple bamboo plants is attributed to two possible pathways. One was a block in chlorophyll biosynthesis, which led to the failure in biosynthesis of the thylakoid membrane. The other one was a disruption in chloroplast development. The lack of thylakoid membrane may have inhibited the conversion of coproporphyrinogen III (Coprogen III) to protoporphyrin IX (Proto IX) during the chlorophyll biosynthesis because the enzyme responsible for this conversion, protogen oxidase, is bound to the thylakoid membrane. The abnormalities in chloroplasts and a low concentration of chlorophyll in the variegated leaves led to a significantly lower photosynthetic rate than in the entirely green leaves, as demonstrated in the light-response curve.
\end{abstract}

Bamboo is an important ornamental plant. Among different bamboo cultivars, those with variegated leaves have recently gained popularity because of their characteristic striped patterns on the leaves. Variegated temple bamboo is a variegated species from Xiamen, Fujian Province, China (Chen and Wang, 2005; Yi et al., 2007). The leaves of the variegated species have white or yellow stripes, and sometimes an entire leaf is white. Moreover, the culm sheath is green with longitudinal, white or yellow stripes. However, the color phenotypes are unstable and may vary according to the cultivation conditions and/or the leaf developmental stage. Although this type of variability is reported among other bamboo species such as Pseudosasa japonica 'Akebonosuji' (Jiang et al., 2016), Pleioblastus kongosanensis f. aureo-striatus, and Hibanobambusa tranquillans f. shiroshima (Wang et al., 2012), the underlying mechanism for the presence of striped leaves in bamboo remains unclear. Plants with variegated leaves provide suitable materials

Received for publication 29 Jan. 2018. Accepted for publication 19 Mar. 2018. This work was supported by the Education Scientific Research Project for Middle-aged and Young Teachers of Fujian Province [JAT170197], the Central Fiscal Forestry Science and Technology Extension Project [2013, No. 7], the Science and Technology Major Projects of Fujian Province [2013NZ0001], the Regional Development Project of Fujian Province [2015N3015].

${ }^{1}$ Corresponding author. E-mail: zys1960fj@163.com. for studying the mechanism of chlorophyll biosynthesis and biodegradation, the chloroplast structure, and the photosynthetic process.

The biosynthesis of chlorophyll is a complex process, which involves chemical changes that occur in four steps. In the first step, glutamic acid is converted to 5-aminolevulinic acid (ALA) and two molecules of ALA form a single molecule of pyrrole porphobilinogen (PBG). In the second step, PBG is isomerized to uroporphyrinogen III (Urogen III), which, in turn, is decarboxylated to Coprogen III. Coprogen III is cyclized and oxidized to Proto IX, which chelates $\mathrm{Mg}^{2+}$ to form $\mathrm{Mg}$ protoporphyrin (Mg-Proto), and Mg-Proto is methylated and cyclized to monobinyl protochlorophyllide (Pchlide) a. In the third step, monobinyl Pchlide a is transformed to chlorophyllide a through oxidoreductase catalysis of Pchlide, an intermediate compound formed in this step. In the last step, chlorophyll a is synthesized by propionic acid esterification between the tetracyclic ring of chlorophyllide a and a phytol tail (Pan, 2012). A blockage at any of these steps will stop the biosynthesis of chlorophyll.

Chloroplast is the site of photosynthetic reactions. Changes in chloroplast structure of variegated mutants are caused by defects in chlorophyll biosynthesis (Nakanishi et al., 2005). An important process in chloroplast development is the formation of the thylakoid system. The stacked membranous structure of chloroplasts that contains chlorophyll is an important site for 
photosystem II (PSII) reactions. Insufficient photosynthetic membrane proteins lead to low net photosynthetic rates (Bertamini and Nedunchezhian, 2003; Snider et al., 2015). The formation of the thylakoid system depends on the combined activities of chloroplast- and nuclear-encoded proteins (Ulrich et al., 2004). If there are any blockages or mutations in this process, defective chloroplasts are produced which are characterized by disorganized thylakoid membranes (Lv et al., 2010; Xia et al., 2015; Zhu et al., 2016). A number of chlorophyll-less mutants have been identified in Zea mays (Lonosky et al., 2004), Glycine max (Stockinger and Walling, 1994), Triticum aestivum (Cao et al., 2006), Hordeum vulgare var. Prato (Preiss and Thornber, 1995), Arabidopsis thaliana (Carol et al., 1999), and Oryza sativa (Jung et al., 2003).

In this study, we conducted a series of experiments to examine the relationship among the photosynthetic pigments, chloroplast structure, and photosynthesis light-response curve in different phenotypes of variegated temple bamboo to understand the differences in leaf color and to explore the relationship among photosynthetic pigments, chloroplast structure, and photosynthetic physiology. We quantified the chlorophyll, carotenoids, and chlorophyll biosynthetic precursors; observed the microstructure and ultrastructure of leaves; and fitted the light-response curves of variegated temple bamboo.

\section{Materials and Methods}

Plant materials and growth conditions. Twenty-twoyear-old variegated temple bamboo plants propagated from root cuttings were bought from a local nursery on 10 Mar. 2016. All plants were transplanted into strip planting grooves $(60 \mathrm{~cm}$ wide, $3 \mathrm{~m}$ long, and $50 \mathrm{~cm}$ deep) with $1 \mathrm{~m}$ between plants using a mixture of sand and topsoil $(1: 1 \mathrm{v} / \mathrm{v})$ and were grown under natural conditions at the Bamboo Research Institute, Fujian Agriculture and Forestry University, Fuzhou, Fujian Province, China (lat. $26^{\circ} 5^{\prime} \mathrm{N}$, long. $119^{\circ} 13^{\prime} \mathrm{E}$, elevation $12 \mathrm{~m}$ ), where the average solar radiation per year is $\approx 1246 \mathrm{~kW} \cdot \mathrm{h} \cdot \mathrm{m}^{-2}$. The average air temperature during the growing season (from March to August) was $27.6{ }^{\circ} \mathrm{C} / 19.7{ }^{\circ} \mathrm{C}$ (day/ night), and the relative humidity was between $50 \%$ and $65 \%$. Plants were grown under natural light. The frequency of watering the plants depended on rainfall and temperature, ensuring that plants had a sufficient water supply. Each plant was applied with $0.5 \mathrm{~kg}$ base manure with $45 \%$ organic material $(0.8 \mathrm{~N}-0.3 \mathrm{P}-$ $0.4 \mathrm{~K}$, granular biological organic fertilizer; Nengliangdan, Hebei, China) while spraying $200 \mathrm{~mL}$ of $5 \mathrm{~g} \cdot \mathrm{L}^{-1}$ compound fertilizer $(10 \mathrm{~N}-$ 3.5P-5.8K, water-soluble compound fertilizer; Nengliangdan) every $15 \mathrm{~d}$ from May to August.
The entirely green leaves, entirely yellow-white leaves, and striped leaves were randomly distributed and could be found on each plant of variegated temple bamboo. According to the level of variegation on the adaxial surface, the foliage was divided into four phenotypes (Fig. 1): phenotype A (entirely green), phenotype B (green leaf marked with yellow-white or yellow-green stripes with the green area $\geq 50 \%$ of the whole leaf area), phenotype $\mathrm{C}$ (yellow-white leaf with green or yellow-green stripes with the yellow-white area $>50 \%$ ), and phenotype D (entirely yellowwhite). Leaves in second apical layer were sampled randomly during the experimental trial between June and Aug. 2016.

Assessment OF CHLOROPHYLl AND CAROTENOID CONTENT. A mixed liquid extraction method (Lichtenthaler, 1987), with a solution of acetone and ethanol $(1: 1, \mathrm{v} / \mathrm{v})$, was used to determine the chlorophyll content. For each leaf phenotype, three fresh leaves were sampled randomly from each plant, and then cut into $0.1 \times 0.1 \mathrm{~cm}$ pieces. Samples of the leaf pieces collected from all plants weighed $0.25 \mathrm{~g}$ with three replications per phenotype. Then, we immersed each sample in $25 \mathrm{~mL}$ of acetone: ethanol $(1: 1)$ solution for $48 \mathrm{~h}$ in the dark until all the pieces turned completely white. The chlorophyll content was determined by measuring the optical density (OD) of the liquid extract at 470, 663, and $645 \mathrm{~nm}$ using a spectrophotometer (T6; Persée, Beijing, China). For each sample, three readings of OD were recorded, with 36 readings in total.

The chlorophyll and carotenoid content (milligrams per gram) of each phenotype was calculated as follows (Lichtenthaler and Wellburn, 1985):

$$
C_{\mathrm{a}}=\left(12.72 \times \mathrm{OD}_{663}-2.59 \times \mathrm{OD}_{645}\right) \times V /(1,000 \times W)
$$

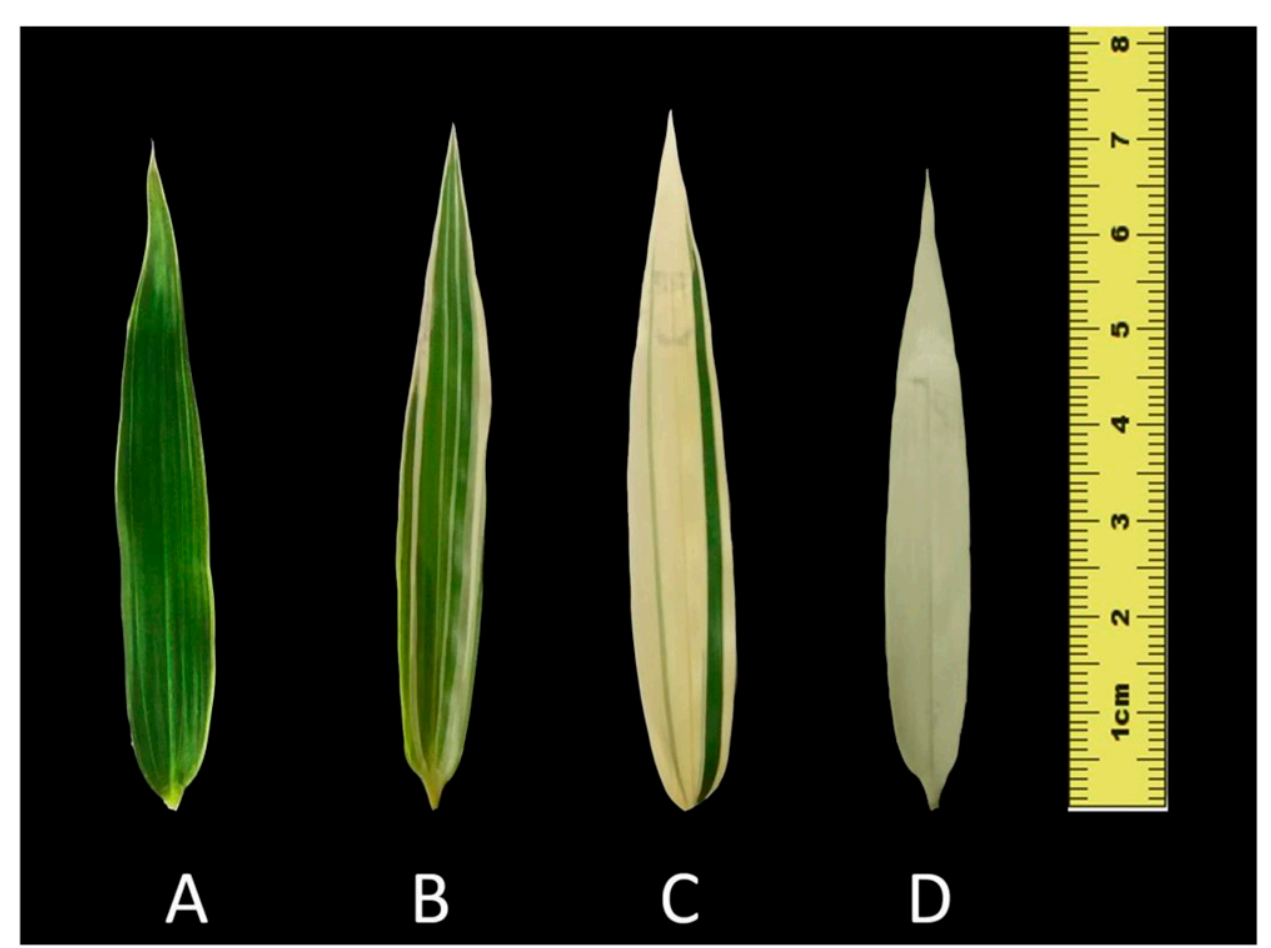

Fig. 1. Four phenotypes of leaves of variegated temple bamboo. Phenotype A, B, C, and D refer to entirely green leaves, green leaves marked with yellow-white or yellow-green stripes area with the green area $\geq 50 \%$ of the whole leaf area, yellow-white leaves with green or yellow-green stripes with the yellow-white area $>50 \%$, and entirely yellow-white leaves, respectively. 


$$
\begin{aligned}
& C_{\mathrm{b}}=22.88 \times \mathrm{OD}_{663}-4.67 \times \mathrm{OD}_{645} \times V /(1,000 \times W) \\
& C_{(\mathrm{a}+\mathrm{b})}=20.29 \times \mathrm{OD}_{663}-8.04 \times \mathrm{OD}_{645} \times V /(1,000 \times W) \\
& \mathrm{CARO}=\left(\mathrm{OD}_{470} \times V / W-2.05 \times C_{\mathrm{a}}-114.8 \times C_{\mathrm{b}}\right) / 245,
\end{aligned}
$$

where $C_{\mathrm{a}}$ is the content of chlorophyll $a(\mathrm{Chl} a), C_{\mathrm{b}}$ is the content of chlorophyll $b(\mathrm{Chl} b), C_{(\mathrm{a}+\mathrm{b})}$ is the chlorophyll $a+b$ $(\mathrm{Chl} a+b)$, CARO is the content of carotenoids, $V$ stands for the volume of liquid extract, and $W$ stands for the weight of one sample.

ASSESSMENT OF BIOSYNTHETIC PRECURSORS OF CHLOROPHYLL. For each phenotype, five fresh leaves were collected randomly from each plant, with 100 leaves per phenotype in total, and then grinded to powder in liquid nitrogen. We collected at least $4.0 \mathrm{~g}$ of mixed powder from each phenotype for the following measurements.

Samples of the mixed leaf powder weighing $1.0 \mathrm{~g}$ with three replications per phenotype were prepared for ALA measurement. ALA was extracted using the method previously described by Zhang et al. (2014) and determined by measuring the OD of the liquid extract at $553 \mathrm{~nm}$.

The concentrations of PBG, Urogen III, and Coprogen III were extracted by the method described by Bogorad (1962). $0.5 \mathrm{~g}$ of mixed leaf powder was used for PBG measurement, whereas $1.0 \mathrm{~g}$ was needed for Urogen III and Coprogen III measurement. The content of three chlorophyll biosynthesis precursors was determined by measuring the OD of the liquid extract at 553, 405.5, and $399.5 \mathrm{~nm}$, respectively.

A $0.5 \mathrm{~g}$ sample of mixed leaf powder was used to measure the concentrations of Proto IX, Mg-Proto, and Pchlide. These three chlorophyll biosynthesis precursors were extracted using the method of Hodgins and Van Huystee (1986) and determined by measuring OD of the liquid extract at 575,590, and $628 \mathrm{~nm}$, respectively.

All measurements were determined using a spectrophotometer (T6). For each sample, three readings of OD were recorded.

Microstructure of Leaves. Samples of cell slices were prepared according to the method of Salgovicová et al. (2007). The structures of the different variegated leaves were observed microscopically after embedding them in paraffin. The four phenotypes were cut into sections of $\approx 0.5 \times 0.5 \mathrm{~cm}$, with three sections per phenotype, then were soaked in FAA solution (50\% ethyl alcohol:formalin:acetic acid at 90:5:5, v/v/v) under vacuum, until there were no bubbles on the leaf surface. The samples were stored at $4{ }^{\circ} \mathrm{C}$ for $24 \mathrm{~h}$. Thereafter, samples were dehydrated in a series of increasing ethyl alcohol concentrations $(50 \%, 70 \%, 85 \%$, and $95 \%)$ and then subjected to two changes of $100 \%$ ethyl alcohol, each for $1 \mathrm{~h}$. The samples were then soaked in two changes of ethyl alcohol (containing 1\% safranin and xylene at $1: 1, \mathrm{v} / \mathrm{v}$ ) and then treated with four changes of $100 \%$ xylene, each for $1 \mathrm{~h}$. The samples were subsequently transferred to a mixture of wax and xylene $(1: 1, \mathrm{v} / \mathrm{v})$ at $37{ }^{\circ} \mathrm{C}$ for $12 \mathrm{~h}$, and then placed in a mixture of wax and xylene $(3: 1, \mathrm{v} / \mathrm{v})$ and maintained at 58 to $60{ }^{\circ} \mathrm{C}$ for $3 \mathrm{~h}$. Thereafter, the samples were incubated four times in $100 \%$ paraffin wax at $60{ }^{\circ} \mathrm{C}$, for $1 \mathrm{~h}$ each time. The samples were embedded in paraffin using a paraffin embedding center (EG1150 H; Leica Biosystems; Wetzlar, Germany), and then cooled. The embedded samples were then sliced into 8-10 $\mu \mathrm{m}$ cross-sections using a microtome (RM2235; Leica Biosystems) and dried at $37{ }^{\circ} \mathrm{C}$ for $12 \mathrm{~h}$. Subsequently, the cross-sections were placed in xylene three times, each time for $30 \mathrm{~min}$, and then sealed onto a microslide using a neutral resin. The sections were observed under a light microscope (CI-L; Nikon, Tokyo, Japan).

Ultrastructure OF LEAVES. Based on the microstructure differences among the samples, we observed that phenotype A (entirely green leaves) and phenotype D (entirely yellow-white leaves) warranted further ultrastructural investigation, as described by Bourett et al. (1999). Three leaves of these two phenotypes were cut into $3 \times 4-\mathrm{mm}$ pieces, and soaked in $2.5 \%$ glutaric dialdehyde solution at $4{ }^{\circ} \mathrm{C}$ for $24 \mathrm{~h}$. The samples were then washed with $0.1 \mathrm{~mol} \cdot \mathrm{L}^{-1}$ phosphate buffer. The washed samples were subsequently fixed with $1 \%$ osmium tetroxide for $2 \mathrm{~h}$, after which they were washed again with $0.1 \mathrm{~mol} \cdot \mathrm{L}^{-1}$ phosphate buffer. Finally, the samples were sequentially dehydrated in $30 \%, 50 \%, 70 \%, 80 \%, 90 \%$, and $100 \%$ acetone before embedding them in resin. The samples were sliced into ultrathin sections using a microtome (RM2235) and observed using a transmission electron microscope (HT7700; Hitachi, Tokyo, Japan).

LighT-RESPONSE CURVES OF PHOTOSYNTHESIS FITTING. For each phenotype, three mature leaves chosen from three randomly selected plants were measured for light-response curves, with 12 leaves in total. Measurements were carried out between 0900 and $1130 \mathrm{HR}$ from 6 to 20 July 2016. A $30 \mathrm{~min}$ photoinduction at $800 \mu \mathrm{mol} \cdot \mathrm{m}^{-2} \cdot \mathrm{s}^{-1}$ was necessary before each measurement. And a photosynthetic photon flux density (PPFD) gradient of $800,600,400,200,150,100,50$, and $0 \mu \mathrm{mol} \cdot \mathrm{m}^{-2} \cdot \mathrm{s}^{-1}$ and a $\mathrm{CO}_{2}$ concentration of $400 \mu \mathrm{mol} \cdot \mathrm{mol}^{-1}$ were used to measure the light-response curve using a portable photosynthesis system (LI-6400XT; LI-COR Biosciences, Lincoln, NE). To show the photoinhibition in the plants, the net photosynthesis rate $\left(P_{\mathrm{n}}\right)$ values at another high PPFD gradient of $800,1200,1550$, and $1800 \mu \mathrm{mol} \cdot \mathrm{m}^{-2} \cdot \mathrm{s}^{-1}$ with the same $\mathrm{CO}_{2}$ concentration were measured as well. The data obtained from the two PPFD gradients measurements were collected together for further analysis. The chamber temperature was $35 \pm 0.5^{\circ} \mathrm{C}$, and the relative humidity was $50 \%$ to $70 \%$. At each $P P F D, 3-5$ min adaptation was required before taking a reading, and readings were repeated five times for each leaf.

The light-response curve of photosynthesis is fundamental to understand the photochemical yield of this process, as well as the relationship between $P P F D$ and $P_{\mathrm{n}}$. There are several models for fitting the I-response curve of photosynthesis, including the "rectangular hyperbola model" (Thornley, 1976), the "nonrectangular hyperbola model" (Thornley, 1976), and the "modified model of rectangular hyperbola" (Ye, 2007). In our preliminary measurements, the "modified model of rectangular hyperbola" highly fitted the data $\left(R^{2}>\right.$ 0.99 ), so this model was used for the light-response curves of photosynthesis.

The light-response curves of photosynthesis were fitted following the modified model of rectangular hyperbola (Ye, 2007) as follows:

$$
P_{\mathrm{n}}=\frac{(1-\beta P P F D)}{(1+\gamma \text { PPFD })}\left(\alpha \text { PPFD }+R_{\mathrm{d}}\right)
$$

where $P_{\mathrm{n}}$ is net photosynthetic rate at the $P P F D, R_{\mathrm{d}}$ is the rate of dark respiration, and $\alpha, \beta$ and $\gamma$ are the coefficients that are independent of $P P F D$, in which $\alpha$ is also the initial slope that 
showed the increasing rate of net photosynthetic rate at very low PPFD, and the units for $\beta$ and $\gamma$ are $\mathrm{m}^{2} \cdot \mathrm{s}^{-1} \cdot \mu \mathrm{mol}^{-1}$. Light use efficiency (LUE) is used to evaluate the ability of plant leaf or canopy to use light energy; with a higher value indicating a stronger ability to use light (Gitelson et al., 2015; Kataria et al., 2013). The value could be caculated as follows (Ye et al., 2017):

$$
\mathrm{LUE}=\alpha \frac{1-\beta \mathrm{PPFD}}{1+\gamma \mathrm{PPFD}}-\frac{R_{\mathrm{D}}}{\mathrm{PPFD}}
$$

Once leaves receive light from dark condition, they will use light to fix $\mathrm{CO}_{2}$ immediately, and the value of LUE will reach the maximum point at very low $P P F D$, then decrease with increasing $P P F D$ (Ye et al., 2017). According to the Eqs. [5] and [6], LUE $=P_{\mathrm{n}} / P P F D$ could be calculated, and the maximum LUE is equal to the initial slope $(\alpha)$. So, $\alpha$ could also indicate the maximum LUE of leaves at very low PPFD.

The light compensation point, LCP, was calculated as follows (Ye et al., 2013):

$$
\mathrm{LCP}=-\frac{R_{\mathrm{d}}}{\alpha}
$$

The light saturation point, LSP, was determined using the formula (Ye et al., 2013):

$$
\operatorname{LSP}=\frac{\sqrt{(\beta+\gamma) / \beta}-1}{\gamma}
$$

The maximum photosynthetic rate, $P_{\mathrm{n}-\max }$, was calculated as follows (Ye et al., 2013):

$$
P_{\mathrm{n}-\max }=\alpha \frac{\sqrt{\beta+\gamma}-\sqrt{\beta}}{\gamma}-R_{\mathrm{d}}
$$

DAta ANALysis. Means \pm SE were calculated and analysis of variance was performed using SPSS statistical software (version 19.0; IBM Corp., Armonk, NY). When significant differences occurred among a measured parameter, means were separated by Duncan's test at $P \leq 0.05$.

Bivariate correlation analysis was used to analyze the relationships between $P_{\mathrm{n}}$ and $\mathrm{Chl} a, \mathrm{Chl} b$, as well as Chl $a+b$ by Spearman's rank correlation test using SPSS statistical software (version 19.0). Each $P_{\mathrm{n}}$ value was matched to the chlorophyll content in relative phenotype, and all the $P_{\mathrm{n}}$ data at different PPFDs was used for these analyses. Graphs were generated using Origin software (version 9.0; OriginLab Corp., Northampton, MA).

\section{Results}

Chlorophyll and CARotenoId CONTENT IN THE DIFFERENT PHENOTYPES. There were significant differences in chlorophyll and carotenoid contents among the four phenotypes $(P<0.01)$, which decreased significantly with the reduction in the percentage of green area. In phenotype $\mathrm{D}$, the contents of Chl $a$, $\mathrm{Chl} b$, and carotenoids were significantly lower than those in the other phenotypes, however, the value of carotenoid/Chl $a+b$ was greater than 1 and significantly higher than other three phenotypes. Chl $a+b$ in the entirely yellow-white leaves (phenotype D) was $0.67 \%$ of that in the entirely green leaves (phenotype A) (Table 1), indicating that the variability in the leaf color among phenotypes could be attributed to the differences in the content of photosynthetic pigments.

CONTENT OF CHLOROPHYLL PRECURSORS IN DIFFERENT PHENOTYPES. The relative concentrations of these precursors in the four phenotypes, with respect to the corresponding values in phenotype A, are shown in Fig. 2. There were no significant differences in ALA content among phenotypes. However, the content of the remaining six precursors showed significant differences $(P<0.05)$. PBG decreased with a decrease in chlorophyll content. This, combined with the high concentrations of precursors Urogen III and Coprogen III indicates the conversion from ALA to PBG in variegated leaves. Urogen III and Coprogen III in phenotype D were significantly higher than in phenotype A, with a relative content of 1.48 and 2.25, respectively. The biosynthesis conversion of Coprogen III to Proto IX was significantly decreased, and the content of Proto IX in phenotype D was only $15 \%$ of that in phenotype A. The fact that Urogen III and Coprogen III contents in phenotype D were higher than in the other phenotypes indicates that chlorophyll biosynthesis was blocked at a site between Coprogen III and Proto IX.

Microstructural and ultrastructural features of MESOPHYLL CELLS. At the microstructural level, the cells of the four phenotypes in the epidermal area were with the same structure. Cells of the adaxial epidermis were elliptical and had a uniform size, with three to five inflated cells forming a fanshaped structure present at $8-12$ cell intervals. The cells of the abaxial epidermis were irregular, and the mesophyll layers consisted of three to five cells. The mesophyll cells of phenotype A were dark in color and had intact organelles (Fig. 3A), whereas phenotype B presented two forms, the one with green stripes had dark variegated cells whereas the one with yellow-green stripes had one to two layers of light variegated cells near the adaxial epidermis (Fig. 3B). The mesophyll cells of phenotype $\mathrm{C}$ also presented two forms, the one with yellow-white stripes had light color cells and the one with green stripes had dark cells (Fig. 3C). The mesophyll cells of phenotype $\mathrm{D}$ were with a light color and characterized by the presence of cavities in the mesophyll tissue (Fig. 3D). On the basis of these observations, we conjectured that the color differences among phenotypes were due to the differences in general organelle structure of the mesophyll cells. To verify this assumption, we determined the mesophyll ultrastructure.

Transmission electron microscopy revealed that the cellular ultrastructure of mesophyll cells in the green leaves of phenotype A remained intact with a compact arrangement of organelles. The cell nucleus, chloroplasts, and vacuoles were clearly visible (Fig. 4A). However, the general trend of structure changes in mesophyll cells of the entirely yellowwhite leaves (phenotype D), was that etioplasts were found instead of intact chloroplasts in most cells (Fig. 4B).

A further observation of the chloroplast structure in the two phenotypes showed that, in the leaves of phenotype A, the elliptical chloroplasts retained structural integrity with folded thylakoid membranes. The granum thylakoid was clearly visible and osmiophilic granules were dispersed in the plastids (Fig. 4C). By contrast to the green leaves, the preserved chloroplasts in the phenotype D leaves showed a degraded condition, including those with unconsolidated thylakoids and 
Table 1. Content of chlorophyll $a(\mathrm{Chl} a)$, chlorophyll $b(\mathrm{Chl} b)$, chlorophyll $a+b(\mathrm{Chl} a+b)$, carotenoid and carotenoid/Chl $a+b$ in leaves of different phenotypes of variegated temple bamboo. Phenotypes A, B, C, and D refer to entirely green leaves, green leaves marked with yellowwhite or yellow-green stripes area with the green area $\geq 50 \%$ of the whole leaf area, yellow-white leaves with green or yellow-green stripes with the yellow-white area $>50 \%$, and entirely yellow-white leaves, respectively.

\begin{tabular}{llcccc}
\hline & Chl $a\left(\mathrm{mg} \cdot \mathrm{g}^{-1}\right)$ & Chl $b\left(\mathrm{mg} \cdot \mathrm{g}^{-1}\right)$ & Chl $a+b\left(\mathrm{mg} \cdot \mathrm{g}^{-1}\right)$ & Carotenoid $\left(\mathrm{mg} \cdot \mathrm{g}^{-1}\right)$ & Carotenoid/Chl $a+b$ \\
\cline { 2 - 6 } Phenotype & $1.776 \pm 0.017 \mathrm{a}^{\mathrm{z}}$ & $0.335 \pm 0.004 \mathrm{a}$ & $2.111 \pm 0.021 \mathrm{a}$ & $0.414 \pm 0.004 \mathrm{a}$ & $0.196 \pm 0.001 \mathrm{~b}$ \\
A & $1.203 \pm 0.011 \mathrm{~b}$ & $0.206 \pm 0.002 \mathrm{~b}$ & $1.410 \pm 0.011 \mathrm{~b}$ & $0.363 \pm 0.005 \mathrm{~b}$ & $0.258 \pm 0.001 \mathrm{~b}$ \\
B & $0.493 \pm 0.035 \mathrm{c}$ & $0.088 \pm 0.003 \mathrm{c}$ & $0.581 \pm 0.006 \mathrm{c}$ & $0.182 \pm 0.001 \mathrm{c}$ & $0.314 \pm 0.003 \mathrm{~b}$ \\
C & $0.011 \pm 0.001 \mathrm{~d}$ & $0.004 \pm 0.002 \mathrm{~d}$ & $0.0142 \pm 0.004 \mathrm{~d}$ & $0.024 \pm 0.0002 \mathrm{~d}$ & $2.018 \pm 0.412 \mathrm{a}$ \\
D & &
\end{tabular}

${ }^{\mathrm{z}}$ Within the same row, different lowercase letters indicate significant differences between the means $(n=3)$ at $P<0.05$ using Duncan's test.

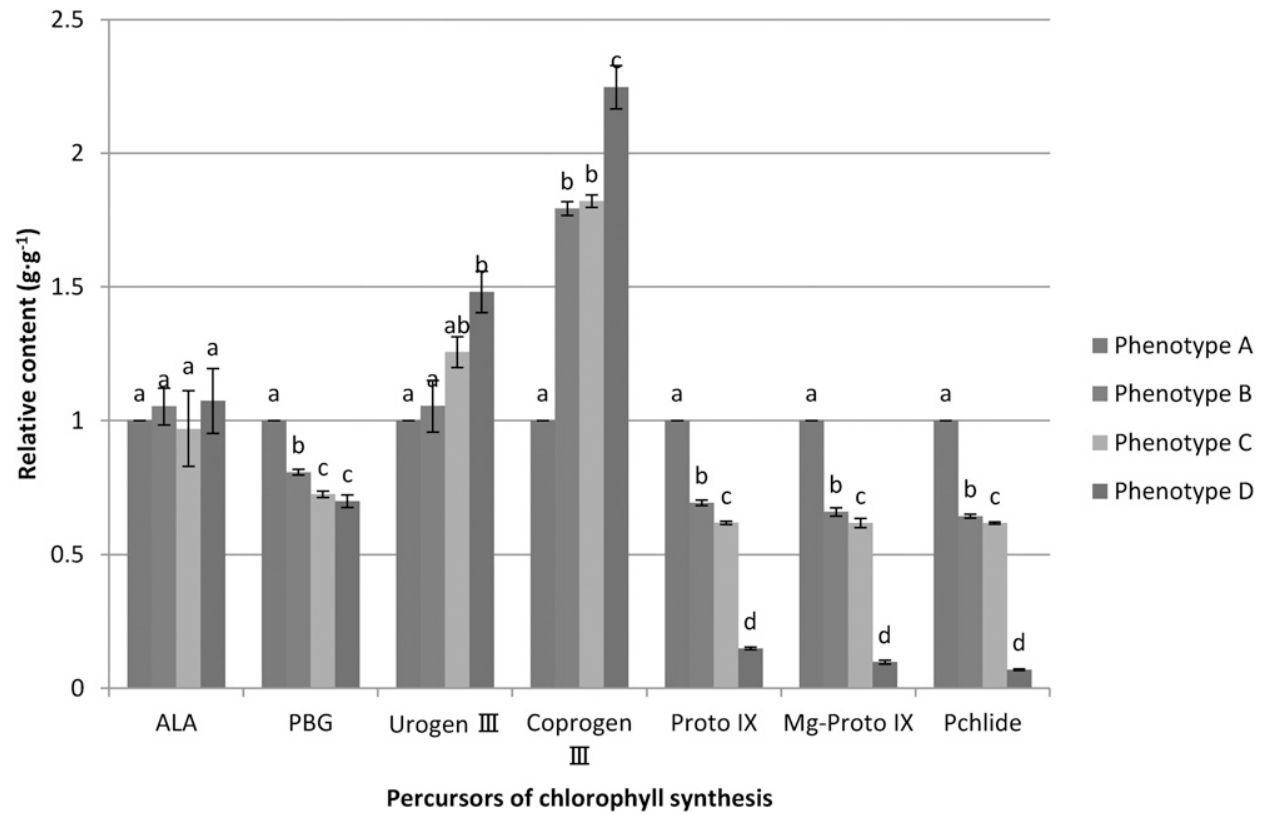

Fig. 2. Variation in the contents of chlorophyll biosynthesis precursors in the different variegated leaves of variegated temple bamboo. Phenotype A, B, C, and D refer to entirely green leaves, green leaves marked with yellow-white or yellow-green stripes with the green area $\geq 50 \%$ of the whole leaf area, yellow-white leaves with green or yellow-green stripes with the yellow-white area $>50 \%$, and entirely yellow-white leaves, respectively. Duncan grouping was made within each precursor [mean $\pm \operatorname{SE}(n=3)]$, where the same lowercase letter appears above the vertical bar, values do not differ significantly at $P<0.05$. $\mathrm{PBG}=$ pyrrole porphobilinogen; ALA $=$ 5-aminolevulinic acid; Urogen III = uroporphyrinogen III; Coprogen III = coproporphyrinogen III; Proto IX = protoporphyrin IX; Mg-Proto $=$ Mg-protoporphyrin; Pchlide $=$ protochlorophyllide .

fragmented or no chloroplast membranes (Fig. 4D and E). Furthermore, we were unable to detect a clear folded thylakoid membrane (the site of PSII) in these cells.

LIGHT-RESPONSE CURVES OF PHOTOSYNTHESIS OF VARIEGATED TEMPLE BAMBOo. Chlorophyll is the most important photosynthetic pigment in plants, and its concentration directly affects photosynthetic parameters. The modified model of rectangular hyperbola came quite close to the measured values for phenotypes A, B, and C with adjusted $R^{2}$ values of 0.999 , 0.997 , and 0.993 , respectively (Table 2 ). However, the net photosynthetic rate of the entirely yellow-white leaves (phenotype D) was consistently less than zero (Fig. 5); therefore, it could not fit well the modified model of rectangular hyperbola, and the adjusted $R^{2}$ was only 0.327 (Table 2). The lightresponse curves of phenotypes A, B, and C are shown in Fig. 5. The overall net photosynthetic rate increased with increasing chlorophyll content. The photosynthetic rate increased rapidly with the PPFD, reached the LSP, and decreased afterward because of photoinhibition. According to the modified model of rectangular hyperbola, the $P_{\text {n-max }}$ and LSP decreased for phenotypes $\mathrm{A}, \mathrm{B}$, and $\mathrm{C}$ whereas the LCP increased, and the dark respiration rate was not significantly different among the three groups (Table 2), which indicated a positive correlation between the chlorophyll content and net photosynthetic rate (Table 3). The $\alpha$ value of phenotype $\mathrm{A}$ and $\mathrm{B}$ are significantly greater than the $\alpha$ value of phenotype $C$, whereas the $\alpha$ value of phenotype B was higher than that of phenotype A (Table 2). It indicated a significant higher LUE at very low PPFD of phenotype $\mathrm{A}$ and $\mathrm{B}$ than phenotype $\mathrm{C}$.

\section{Discussion}

Chlorophyll biosynthesis is a complex process of chemical changes that involves four stages, 15 reactions, and 15 related enzymes (Wang et al., 2009). In the presence of any barrier blocking this pathway, chlorophyll biosynthesis is ended. Studies on these blocking steps have focused on leaf color mutants or plants subjected to stress, and most of these studies indicated that different sites in the biosynthetic process were blocked in different plant species. A block between PBG and Urogen III was detected in Oncidium mutants (Tian et al., 2015) and Brassica napus (Sun et al., 2007) under salt stress, whereas a block between Urogen III and Coprogen III was observed in a Brassica juncea mutant under cadmium stress (Lv et al., 2010). A block between Proto IX and Mg-Proto was observed in an O. sativa mutant (Belyaeva and Litvin, 2009). All these studies showed that if one step was blocked in the process of chlorophyll biosynthesis, the concentration of the precursor upstream of the block site would increase, whereas the concentration of intermediates downstream of the blockage would decrease. Under such circumstances, lower amounts of chlorophyll would be synthesized, and the leaves of plants would turn white or yellow. In variegated temple bamboo plants, we classified leaves into four types based on color or level of variegation, and observed significant differences in the content of photosynthetic pigments among all phenotypes. In our study, the measured 

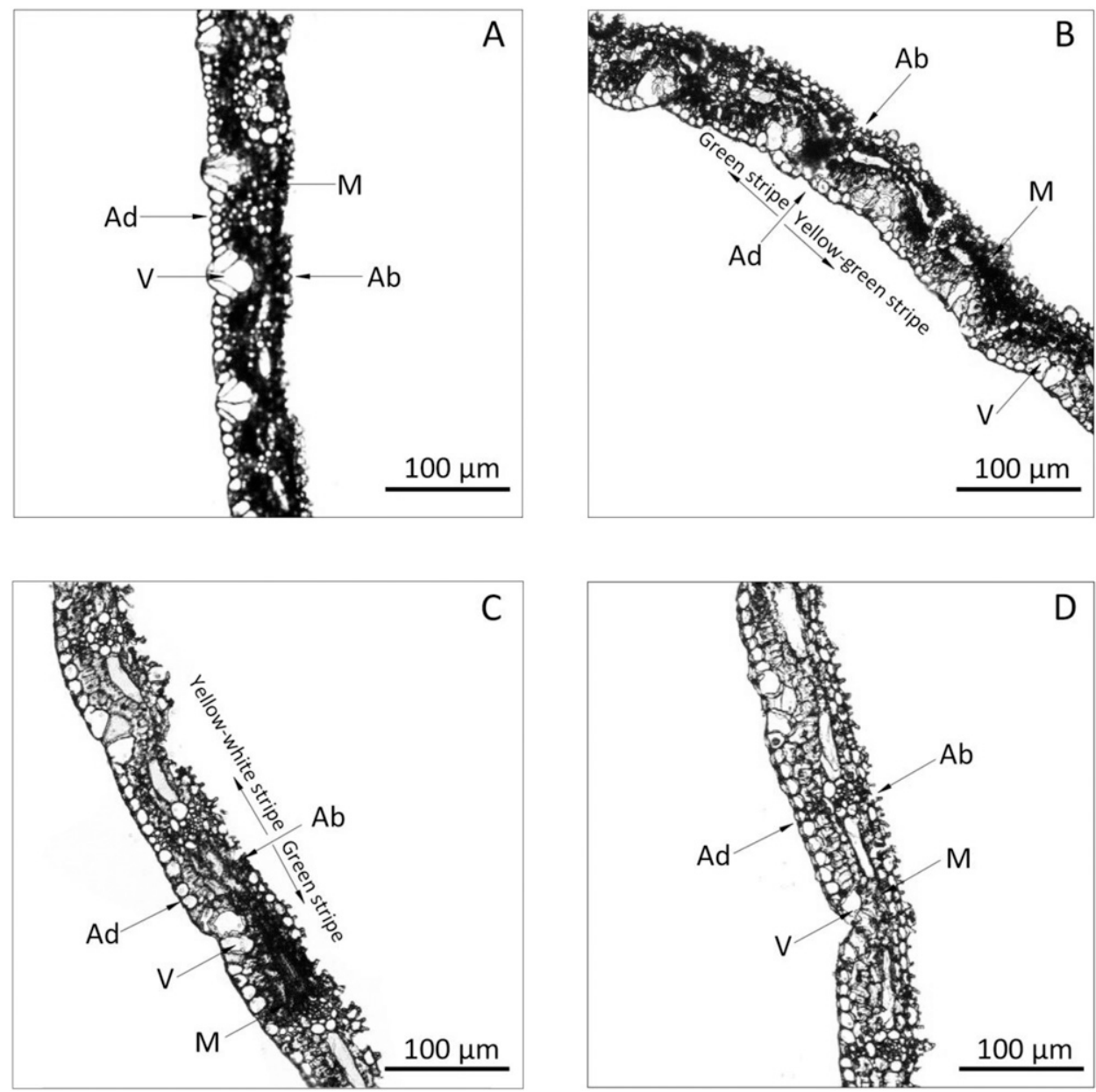

Fig. 3. Microstructure of the different variegated leaves of variegated temple bamboo: (A) mesophyll microstructure in entirely green leaves (phenotype A); (B) mesophyll microstructure in green leaves with yellow-white stripes (phenotype B); (C) mesophyll microstructure in yellow-white leaves with green stripes (phenotype C); (D) mesophyll microstructure in entirely yellow-white leaves (phenotype D). Ad = adaxial epidermis; $\mathrm{Ab}=$ abaxial epidermis; $\mathrm{M}=$ mesophyll; $\mathrm{F}$ = fan-shaped structure.

concentrations of specific chlorophyll biosynthesis precursors suggest a blockage site between Coprogen III and Proto IX. This result is similar to Ananas comosus var. bracteatus with complete white leaves, where the enzyme activity of porphobilinogen deaminase and uroporporphyrinogn III synthase, that catalyze the transition of PBG to Urogen III, was significantly decreased ( $\mathrm{Li}$ et al., 2017). The rapid decrease of Proto IX concentration lead to a correspondingly lower amount of photosynthetic pigment in phenotypes $\mathrm{B}, \mathrm{C}$, and $\mathrm{D}$ than in phenotype A. The Proto IX is the major branch point in biosynthesis between chlorophyll and heme or other plant pigments. And chelatase enzymes play an important role in this biosynthesis progress. Insertion of $\mathrm{Fe}^{2+}$ into this porphyrin macrocycle with the catalysis of ferrochelatase leads to heme and phytochromobilin, whereas insertion of $\mathrm{Mg}^{2+}$ with the catalysis of Mg-chelatase is the first step on the chlorophyll branch. This means the two different chelatases must compete for Proto IX at this branch point (Cornah et al., 2003). Therefore, the low concentration of Proto IX resulted in the striped and entirely yellow-white phenotype in variegated temple bamboo.

The suppressed chlorophyll biosynthesis in the yellow-white part of variegated temple bamboo leaves associated with an abnormal chloroplast structure, especially in the thylakoid membranes. These features are similar to those observed in stressed plants. Previous studies have shown that if there is a blockage in the chlorophyll biosynthesis pathway, abnormalities occur in the chloroplast structure because of the destruction of membrane system (Lv et al., 2010). In this regard, a high concentration of $\mathrm{Pb}$ has been shown to decrease the chlorophyll content in Robinia pseudoacacia seedlings, where the thylakoids arrangement became disorderly or they disappeared (Zhang et al., 2006). However, we detected osmiophilic granules dispersed in the plastids, indicating that these cellular components may participate in the biosynthesis of thylakoid membranes, and this would provide a basis for the transition from white to green leaves (Zhou et al., 2017). So the relationship between biosynthesis of chlorophyll and thylakoid membranes should be associated with the striped and entirely yellow-white phenotypes.

On one hand, the suppressed chlorophyll synthesis would be due to the failure of proplastids to develop into chloroplasts (Semenova et al., 2017). The light-harvesting Chl $a / b$-binding protein (LHCP) is one of the major protein constituents of the thylakoid membrane of chloroplasts. However, this protein is not detectable among the membrane polypeptides of etioplasts (Apel et al., 1983). Etioplasts do not contain prolamellar bodies and are likely proplastids, which did not develop into chloroplasts (Semenova et al., 2017). In white parts of the leaf of variegated temple bamboo, the low concentration of Chl $a$ and $\mathrm{Chl} b$ is due to the lack of LHCP, which lead to the appearance of etioplasts in mesophyll cells. On the other hand, chlorophyll biosynthesis spans different parts of chloroplast. Urogen decarboxylase converts Urogen III to Coprogen III and coprogen oxidase oxidizes Coprogen III to Protogen IX. In this process, the enzyme catalyzing the oxidation of Protogen IX to Proto IX is bound to the envelope and thylakoid membrane (Manohara and Tripathy, 2000). Because of the degraded condition of thylakoid membranes in the chloroplast, there was no site for the conversion from Urogen III to Proto IX in variegated temple bamboo. However, the present study could not show which pathway was right, so further research at the molecular level is needed.

In addition, primary distribution of carotenoid was on the thylakoid membranes of chloroplasts as well (Pan, 2012), so the degraded membrane system also affected the distribution of carotenoid in variegated temple bamboo. Carotenoid could not only collect and transfer light energy, but also take an important role in photoprotection against photo-oxidation in plants (Pan, 

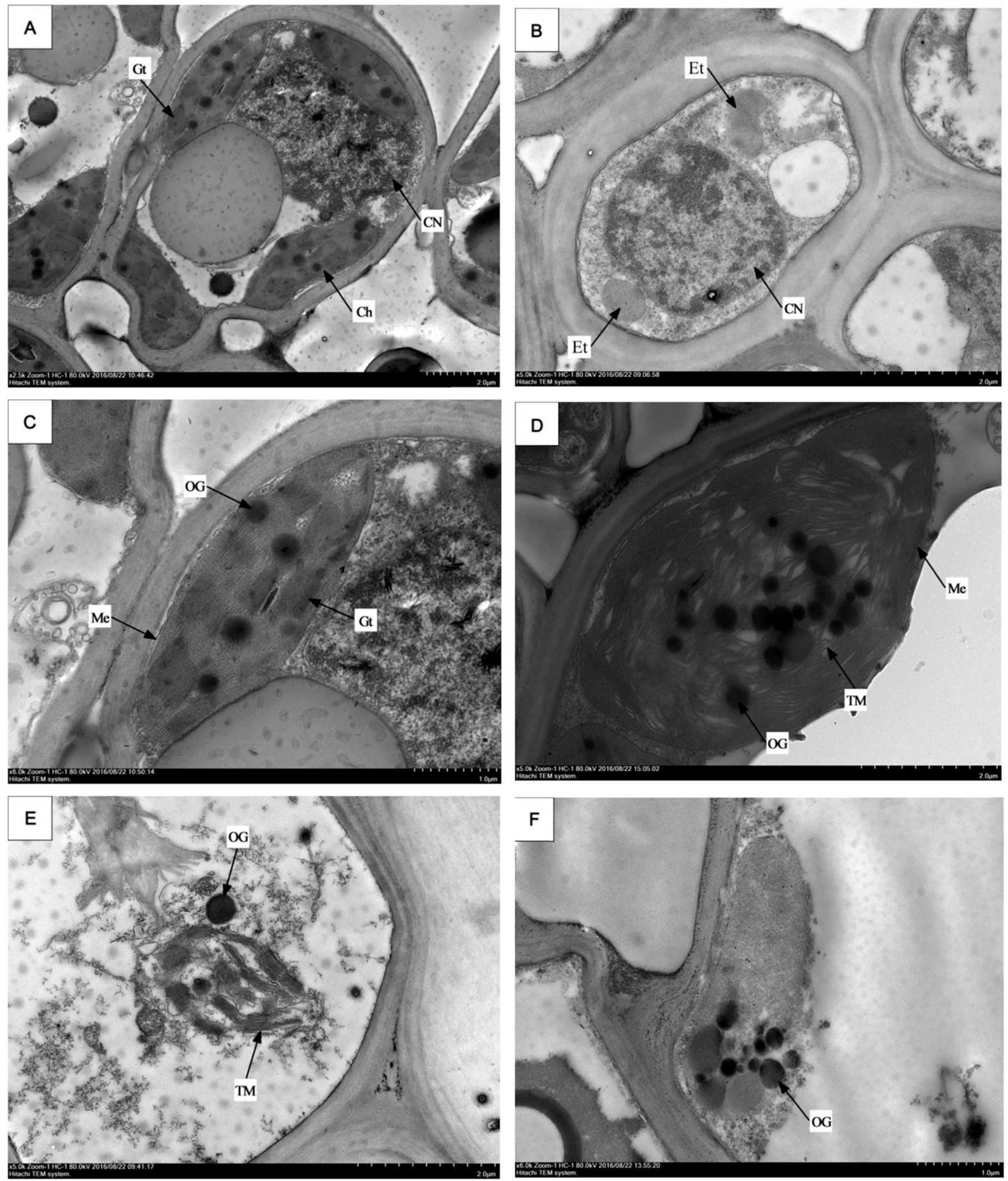

Fig. 4. Mesophyll ultrastructure in different variegated leaves of variegated temple bamboo: (A) complete mesophyll cell in entirely green leaf (phenotype A);

(B) a cell lacking chloroplasts in entirely yellow-white leaf (phenotype D); (C) a normal chloroplast; (D-F) degraded chloroplasts; (D) an unconsolidated thylakoid; (E) an irregular chloroplast; $(\mathbf{F})$ osmiophilic granules in an irregular chloroplast. $\mathrm{Me}=$ chloroplast membrane; $\mathrm{Ch}=\mathrm{chloroplast;} \mathrm{Gt}=\mathrm{granum}$ thylakoid; $\mathrm{OG}=$ osmiophilic granule; $\mathrm{CN}=$ cell nucleus; $\mathrm{Et}=$ etioplast; $\mathrm{TM}=$ thylakoid membrane. 
Table 2. Photosynthetic parameters in the four phenotypes of variegated temple bamboo leaves. The photosynthetic parameters are: initial slope $(\alpha)$, maximum photosynthetic rate $\left(P_{\mathrm{n}-\mathrm{max}}\right)$, light saturation point (LSP), light compensation point (LCP), rate of dark respiration $\left(R_{\mathrm{d}}\right)$. The adjusted $R^{2}$ are presented to show the degree of fit between the calculated and measured values. Phenotypes A, B, C, and D refer to entirely green leaves, green leaves marked with yellow-white or yellow-green stripes area with the green area $\geq 50 \%$ of the whole leaf area, yellowwhite leaves with green or yellow-green stripes with the yellow-white area $>50 \%$, and entirely yellow-white leaves, respectively.

\begin{tabular}{|c|c|c|c|c|c|c|}
\hline & $\alpha\left(\mu \mathrm{mol} \cdot \mu \mathrm{mol}^{-1}\right)$ & $\begin{array}{c}P_{\mathrm{n}-\max } \\
{\left[\mathrm{CO}_{2}\left(\mu \mathrm{mol} \cdot \mathrm{m}^{-2} \cdot \mathrm{s}^{-1}\right)\right]}\end{array}$ & $\begin{array}{c}\text { LSP } \\
\left(\mu \mathrm{mol} \cdot \mathrm{m}^{-2} \cdot \mathrm{s}^{-1}\right) \\
\end{array}$ & $\begin{array}{c}\mathrm{LCP} \\
\left(\mu \mathrm{mol} \cdot \mathrm{m}^{-2} \cdot \mathrm{s}^{-1}\right) \\
\end{array}$ & $\begin{array}{c}R_{\mathrm{d}} \\
{\left[\mathrm{CO}_{2}\left(\mu \mathrm{mol} \cdot \mathrm{m}^{-2} \cdot \mathrm{s}^{-1}\right)\right]}\end{array}$ & $\begin{array}{c}\text { Adjusted } \\
\text { factor }\left(R^{2}\right)\end{array}$ \\
\hline Parameter & \multicolumn{6}{|c|}{ mean $\pm \mathrm{SE}$} \\
\hline Phenotype A & $0.074 \pm 0.0031 \mathrm{~b}^{\mathrm{z}}$ & $8.05 \pm 0.144 b$ & $603.66 \pm 24.682 b$ & $7.63 \pm 1.142 \mathrm{a}$ & $0.57 \pm 0.093 \mathrm{a}$ & $0.999 \pm$ \\
\hline Phenotype C & $0.018 \pm 0.0017 \mathrm{a}$ & $1.00 \pm 0.118 \mathrm{a}$ & $431.86 \pm 70.416 \mathrm{a}$ & $27.75 \pm 3.250 \mathrm{~b}$ & $0.54 \pm 0.048 \mathrm{a}$ & $0.993 \pm 0.032 \mathrm{a}$ \\
\hline Phenotype D & $-^{\mathrm{y}}$ & - & - & - & - & $0.327 \pm 0.045 \mathrm{~b}$ \\
\hline
\end{tabular}

${ }^{\bar{z}}$ Within the same row, different lowercase letters indicate significant differences between the means $(n=5)$ at $P<0.05$ using Duncan's test. ${ }^{\mathrm{y}}$ The adjusted factor $\left(R^{2}\right)$ of phenotype $\mathrm{D}$ showed that the modified model of rectangular hyperbola deviated greatly from the measured values and the fitted values of $\alpha, P_{\mathrm{n}-\max }, \mathrm{LSP}, \mathrm{LCP}$, and $R_{\mathrm{d}}$ are not valid, so no values are shown there.

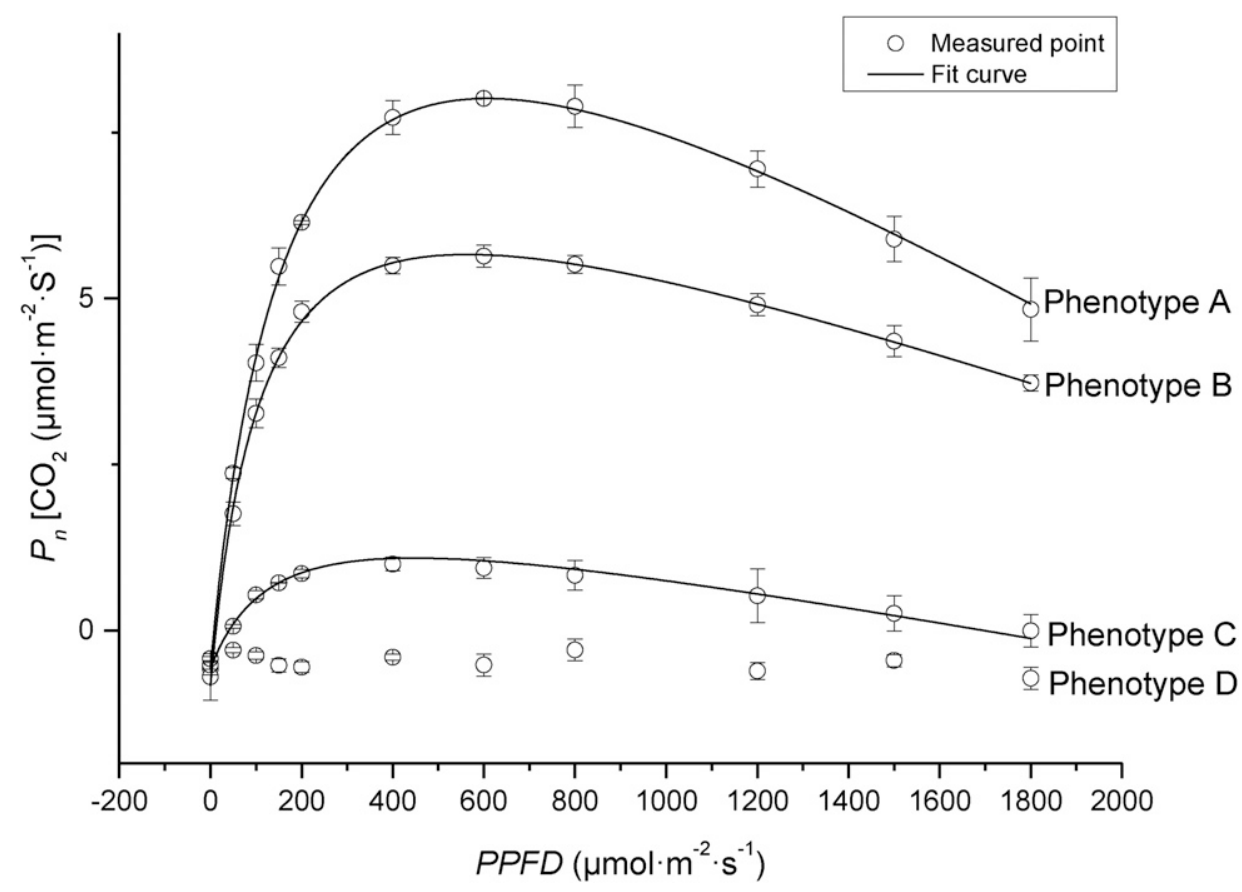

Fig. 5. Response of net photosynthetic rate $\left(P_{\mathrm{n}}\right)$ of variegated temple bamboo to different photosynthetic photon flux density $(P P F D)$ levels. Phenotype A, B, C, and D refer to entirely green leaves, green leaves marked with yellow-white or yellow-green stripes area with the green area $\geq 50 \%$ of the whole leaf area, yellow-white leaves with green or yellow-green stripes with the yellow-white area $>50 \%$, and entirely yellow-white leaves, respectively. The net photosynthetic rate of phenotype $\mathrm{D}$ was consistently less than zero, and the measured value could not fit well the modified model of rectangular hyperbola [adjusted factor $\left(R^{2}\right)$ is 0.327 ], so no fit curve is shown. Vertical bars in the figure indicate means $\pm \operatorname{SE}(n=3)$.

Table 3. Bivariate correlation analysis between net photosynthesis rate $\left(P_{\mathrm{n}}\right)$ and chlorophyll $a(\mathrm{Chl} a)$, chlorophyll $b(\mathrm{Chl} b)$, as well as chlorophyll $a+b(\mathrm{Chl} a+b)$, respectively, using Spearman's rank correlation test. All the $P_{\mathrm{n}}$ data at different photosynthetic photon flux densities (PPFDs) were used for this analysis.

\begin{tabular}{lccc}
\hline Variables & Chl $a$ & Chl $b$ & Chl $a+b$ \\
\hline$P_{\mathrm{n}}$ & $0.762^{* * \mathrm{z}}$ & $0.821^{* *}$ & $0.826^{* *}$
\end{tabular}

${ }^{\mathrm{z}}$ The $* *$ indicated a significant positive correlation between any two variables at the 0.01 level (two-tailed) because all the correlation values are positive.

2012; Sun et al., 2011). In variegated temple bamboo, the concentration of carotenoid in phenotype $\mathrm{D}$ was higher than chlorophyll $a+b$, indicating a stronger photoprotective function.
The malformation of the chloroplast structure as well as the block in chlorophyll biosynthesis together promoted changes in the physiological processes, especially in the photosynthesis process. Previous research has shown that chlorophyll synthesis genes would affect the expression of photosynthesis related genes by the expression of plastid signal (Nakanishi et al., 2005; Wu et al., 2007; Zhang et al., 2006). A missense mutation of $O$. sativa Chldeficient mutant (ygll) in chlorophyll synthesis enzyme affected the expression of many nuclear genes. For example, the expression of $c a b 1 R$, which encodes the LHCP of PSII, was severely suppressed by the $y g l l$ mutation (Wu et al., 2007). The lower photosynthesis rate in variegated phenotypes was significant in variegated temple bamboo. The net photosynthesis rate of different phenotypes decreased significantly with the decrease in chlorophyll concentration. Because measurements were made under identical conditions and there were no significant differences in the number of cell layers in four phenotypes (Fig. 3A-D), the changing trend of measured chlorophyll concentration in four phenotypes could reflect the chlorophyll concentration trend of unit leaf area in different phenotypes. Net photosynthesis was influenced by the chlorophyll content in unit leaf area, which means net photosynthesis was associated with chlorophyll concentration in four phenotypes. According to bivariate correlation analysis, there was a positive correlation between the net photosynthesis rate and chlorophyll concentration (Table 3 ). The net photosynthetic rate of the entirely yellowwhite leaves (phenotype D) was consistently less than zero, indicating a greater respiration rate compared with the photosynthesis rate. We observed significant differences between the LUE at very low PPFD in phenotypes A, B, and phenotype $\mathrm{C}$ (Table 2), which indicated that the phenotypes with higher 
chlorophyll content had a stronger ability to use light at very low $P P F D$ because leaves with more chlorophyll will absorb a higher fraction of the incident light and thus have more excitation energy to drive photosynthesis.

\section{Conclusions}

There were two possible pathways related to the discoloration in the leaves of variegated temple bamboo plants. One possible pathway is that a chlorophyll biosynthesis blockage led to the failure of thylakoid membrane biosynthesis. So there were etioplasts as well as abnormal grana lamellae in yellowwhite part of leaf mesophyll cells. The other possible pathway was that the lack of thylakoid membranes dampened the conversion of Coprogen III to Proto IX because of the biosynthesis failure of the protogen oxidase bound to the thylakoid membrane. These abnormalities in chloroplasts, together with the low concentration of chlorophyll, resulted in a significantly lower photosynthetic rate in the variegated leaves than that of entirely green leaves, which was reflected in the light-response curve. There was a positive correlation between the chlorophyll content and the net photosynthesis rate.

\section{Literature Cited}

Apel, K., I. Gollmer, and A. Batschauer. 1983. The light-depend control of chloroplast development in barley (Hordeum vulgare L.) J. Cell. Biochem. 23:181-189.

Belyaeva, O.B. and F.F. Litvin. 2009. Pathways of formation of pigment forms at the terminal photobiochemical stage of chlorophyll biobiosynthesis. Biochemistry 74:1535-1544.

Bertamini, M. and N. Nedunchezhian. 2003. Photoinhibition of photobiosynthesis in mature and young leaves of grapevine (Vitis vinifera L.). Plant Sci. 164:635-644.

Bogorad, L. 1962. Porphyrin biosynthesis, p. 885-891. In: S.P. Colowick and N.O. Kapha (eds.). Methods in enzymology. Academic Press, New York, NY.

Bourett, T.M., K.J. Czymmek, and R.J. Howard. 1999. Ultrastructure of chloroplast protuberances in rice: Leaves preserved by highpressure freezing. Planta 208:472-479.

Cao, L., H. Wang, D.J. Sun, Y. Feng, X.J. Li, and D.H. Min. 2006. Photobiosynthesis and chlorophyll fluorescence characters of xantha wheat mutants. Xibei Zhiwu Xuebao 26:2083-2087 (in Chinese).

Carol, P., D. Stevenson, C. Bisanz, J. Breitenbach, G. Sandmann, R. Mache, G. Coupland, and M. Kutz. 1999. Mutations in the arabidopsis gene IMMUTANS cause a variegated phenotype by inactivating a chloroplast terminal oxidase associated with phytoene desaturation. Plant Cell 11:57-68.

Chen, S.H. and Z.Z. Wang. 2005. Sinobambusa tootsik Makino ex Nakai var. luteolo-albo-striata S.H. Chen ex Z.Z. Wang, a new variety of Sinobambusa from Xiamen, China. J. Bamboo Res. 24:12 (in Chinese).

Cornah, J.E., M.J. Terry, and A.G. Smith. 2003. Green or red: What stops the traffic in the tetrapyrrole pathway? Trends Plant Sci. 8:224230.

Gitelson, A.A., Y. Peng, and T.J. Arkebauer. 2015. Productivity, absorbed photosynthetically active radiation, and light use efficiency in crops: Implications for remote sensing of crop primary production. J. Plant Physiol. 177:100-109.

Hodgins, R.R. and R.B. Van Huystee. 1986. Rapid simultaneous estimation of protoporphyrin and Mg-porphyrins in higher plants. J. Plant Physiol. 125:311-323.

Jiang, K.Y., M.B. Zhou, H.Y. Yang, and W. Fang. 2016. Cloning and functional characterization of $P j C A O$ gene involved in chlorophyll $b$ biosynthesis in Pseudosasa japonica cv. Akebonosuji. Trees 30:1303-1314.
Jung, K.H., J. Hur, C.H. Ryu, Y. Choi, Y.Y. Chung, A. Miyao, H. Hirochika, and G. An. 2003. Characterization of a rice chlorophylldeficient mutant using the T-DNA gene-trap system. Plant Cell Physiol. 44:463-472.

Kataria, S., K.N. Guruprasad, and S. Ahuja. 2013. Enhancement of growth, photosynthetic performance and yield by exclusion of ambient UV components in $\mathrm{C} 3$ and $\mathrm{C} 4$ plants. J. Photochem. Photobiol. Bol. Biol. 127:140-152.

Li, X., K. Surapathrudu, Y.H. He, X.L. Zhong, S.M. Yu, R.X. Li, L.X. Sun, and J. Ma. 2017. Physiological characterization and comparative transcriptome analysis of white and green leaves of Ananas comosus var. bracteatus. PLoS One 12:e0169838.

Lichtenthaler, H.K. 1987. Chlorophylls and carotenods: Pigments of photosynthetic biomembranes. Methods Enzymol. 148:350-382.

Lichtenthaler, H.K. and A.R. Wellburn. 1985. Determination of total caroteinods and chlorophylls a and b of leaf in different solvents. Biol. Soc. Trans. 11:591-592.

Lonosky, P.M., X. Zheng, V.G. Honavar, D.L. Dobbs, A. Fu, and S.R. Rodermal. 2004. A proteomic analysis of maize chloroplast biogenesis. Plant Physiol. 134:561-574.

Lv, M., H.H. Liu, H.D. Mao, Q.R. Zhao, H.X. Zhao, and S.W. Hu. 2010. Changes of chlorophyll biosynthesis metabolism in chlorophyll-deficient mutant in Brassica juncea. Xibei Zhiwu Xuebao 30:2177-2183 (in Chinese).

Manohara, M.S. and B.C. Tripathy. 2000. Regulation of protoporphyrin IX biosynthesis by intraplastidic compartmentalization and adenosine triphosphate. Planta 212:52-59.

Nakanishi, H., H. Nozue, K. Suzuki, Y. Kaneko, G. Taguchi, and N. Hayashida. 2005. Characterization of the Arabidopsis thaliana mutant $p c b 2$ which accumulates divinyl chlorophylls. Plant Cell Physiol. 46:467-473.

Pan, R.Z. 2012. Plant physiology. High Educ. Press, Beijing, China (in Chinese).

Preiss, S. and J.P. Thornber. 1995. Stability of the apoproteins of lightharvesting complex I and II during biogenesis of thylakoids in the chlorophyll b-less barley mutant Chlorina f2. Plant Physiol. 107:709-717.

Salgovicová, I., Z. Lojanová, and J. Hudák. 2007. Plastid structure and chlorophyll content in leaf primordia of onion bulbs. J. Plant Physiol. 164:19-22.

Semenova, G.A., I.R. Fomina, A.A. Kosobbryukhov, V.Y. Lyubimov, E.S. Nadezhkina, and T.I. Balaknina. 2017. Mesophyll cell ultrastructure of wheat leaves etiolated by lead and selenium. J. Plant Physiol. 219:37-44.

Snider, J.L., D.R. Chastain, C.D. Meeks, G.D. Collins, R.B. Sorensen, S.A. Byrd, and C.D. Perry. 2015. Predawn respiration rates during flowering are highly predictive of yield response in Gossypium hirsutum when yield variability is water-induced. J. Plant Physiol. 183:114-120.

Stockinger, E.J. and L.L. Walling. 1994. A chlorophyll a/b-binding protein gene from soybean (Glycine max [L.] Merr.). Plant Physiol. 104:1475-1476.

Sun, J.Y., N.H. Zhang, and L.F. Du. 2007. Chlorophyll biobiosynthesis in a chlorophyll b-deficient oilseed rape mutant $C r 3529$. Xibei Zhiwu Xuebao 27:1962-1966 (in Chinese).

Sun, X. and T. Wen. 2011. Physiological roles of plastid terminal oxidase in plant stress responses. J. Biosci. 36:951-956.

Thornley, J.H.M. 1976. Mathematical models in plant physiology. Academic Press, London, UK.

Tian, W.W., C.X. Wang, M. Tian, T. Ouyang, and Y. Zhang. 2015. Physiological, biochemical and chlorophyll fluorescence characters of light green stripe mutant in Oncidium. Xibei Zhiwu Xuebao 35:2012-2017 (in Chinese).

Ulrich, E., G. Bernhard, and H. Stefan. 2004. Recent advances in chlorophyll biosynthesis and breakdown in higher plants. Plant Mol. Biol. 56:1-44.

Wang, X.C., X.H. Yue, J. Wu, Q. Liu, and Z.M. Gao. 2012. Appearance and structure analysis of chimeric leaves from two ornamental bamboos. Chinese Agr. Sci. Bul. 28:233-238 (in Chinese). 
Wang, P.R., F.T. Zhang, J.X. Gao, X.Q. Sun, and X.J. Deng. 2009. An overview of chlorophyll biosynthesis in higher plants. Xibei Zhiwu Xuebao 29:629-636 (in Chinese).

Wu, Z.M., X. Zhang, B. He, L.P. Diao, S.L. Sheng, J.L. Wang, X.P. Guo, N. Su, L.F. Wang, L. Jiang, C.M. Wang, H.Q. Zhai, and J.M. Wan. 2007. A chlorophyll-deficient rice mutant with impaired chlorophyllide esterification in chlorophyll biosynthesis. Plant Physiol. 145:29-40.

Xia, X.W., R.Y. Gui, H.Y. Yang, Y. Fu, W. Fang, and M.B. Zhou. 2015. Identification of genes involved in color variation of bamboo culms by suppression subtractive hybridization. Plant Physiol. Biochem. 97:156-164.

Ye, Z.P. 2007. A new model for relationship between irradiance and the rate of photosynthesis in Oryza sativa. Photosynthetica 45:637-640.

Ye, Z.P., D.J. Suggett, P. Robakowki, and H.J. Kang. 2013. A mechanistic model for the photosynthesis-light response based on the photosynthetic electron transport of photosystem II in C3 and C4 species. New Phytol. 199:110-120.

Ye, Z.P., H.L. Zhang, Z.A. Huang, Z.L. Yang, and H.J. Kang. 2017. Model construction of light use efficiency and water use efficiency based on a photosynthetic mechanistic model of light response. Plant Physiol. J. 53:1116-1122 (in Chinese).

Yi, T.P., J.Y. Shi, H.T. Wang, L.S. Ma, and L. Yang. 2007. A new species, some new combinations and new distributions of Bambusodeae, China. J. Sichuan For. Sci. Technol. 28:15-18 (in Chinese). Zhang, F., X.Q. Wan, and J. Zhai. 2014. Effects of nitrogen supplement on chlorophyll biosynthesis and chloroplast ultrastructure of poplar plants under cadmium stress. J. Nuclear Agr. Sci. 28:485-491 (in Chinese).

Zhang, H.T., J.J. Li, J.H. Yoo, S.C. Yoo, S.H. Cho, H.J. Koh, H.S. Seo, and N.C. Paek. 2006. Rice chlorina-1 and chlorina-9 encode ChlD and ChlI subunits of Mg-chelatase, a key enzyme for chlorophyll biosynthesis and chloroplast development. Plant Mol. Biol. 62:325-337.

Zhou, J., Z.P. Jiang, J. Ma, L.F. Yang, and Y. Wei. 2017. The effects of lead stress on photosynthetic function and chloroplast ultrastructure of Robinia pseudoacacia seedlings. Environ. Sci. Pollut. Res. Intl. 24:10718-10726.

Zhu, X.Y., S. Guo, Z.W. Wang, Q. Du, Y.D. Xing, T.Q. Zhang, W.Q. Shen, X.C. Sang, Y.H. Ling, and G.H. He. 2016. Map-based cloning and functional analysis of $Y G L 8$, which controls leaf colour in rice (Oryza sativa). BMC Plant Biol. 16:134. 